Research Paper

\title{
Association between Primary Tumor Location and Prognostic Survival in Synchronous Colorectal Liver Metastases after Surgical Treatment: A Retrospective Analysis of SEER Data
}

\author{
Zheng Wang ${ }^{1 *}$, Xiangyu Wang ${ }^{2 *}$, Ze Zhang ${ }^{2 *}$, Xuan Wang ${ }^{2 *}$, Mo Chen², Lu Lu², Wenwei Zhu², Jubo Zhang ${ }^{2}$, \\ Huliang Jia ${ }^{2}$, Jinhong Chen ${ }^{2}$ \\ 1. Comprehensive Breast Health Center, Ruijin Hospital, Shanghai Jiao Tong University School of Medicine, Shanghai, China \\ 2. Department of General Surgery, Huashan Hospital \& Cancer Metastasis Institute, Fudan University, Shanghai, China \\ *These authors contributed equally to this work. \\ $\triangle$ Corresponding author: Jinhong Chen, PhD\&MD, Department of General Surgery, Huashan Hospital \& Cancer Metastasis Institute, Fudan university, 12 \\ Urumqi Road (M), Shanghai 200040, P.R. China. Tel: \& Fax: +86-21-5288-7172; E-mail: DrHSCJH@outlook.com \\ (c) Ivyspring International Publisher. This is an open access article distributed under the terms of the Creative Commons Attribution (CC BY-NC) license \\ (https://creativecommons.org/licenses/by-nc/4.0/). See http://ivyspring.com/terms for full terms and conditions.
}

Received: 2018.08.16; Accepted: 2019.01.20; Published: 2019.02.26

\begin{abstract}
The prognostic and predictive role of primary tumor location (PTL) in localized and metastatic colorectal cancer (CRC) is a hotspot issue in recent years. However, its prognostic role is still unclear in synchronous colorectal liver metastases (sCRLM), especially in those receiving surgical treatment of primary tumor and liver metastases. Here, a retrospective survival analysis was performed using the Surveillance, Epidemiology, and End Results Program (SEER) database between 2010 and 2014, on patients who were pathologically confirmed SCRLM, and received surgical treatment of both primary tumor and liver metastases. After stringent exclusive procedure, a total of 1508 patients with sCRLM (872 men [57.8\%] and 636 women [42.2\%]; mean age, 60.9 years) were eligible for the final study. Compared with sCRLM with left-sided PTL, cases with right-sided PTL were more likely to be T4 (31.3\% vs. $20.1 \%, \mathrm{p}<0.001)$, N2 $(42.5 \%$ vs. $31.8 \%, \mathrm{p}<0.001)$ and poorly differentiated $(30.5 \%$ vs. $15.1 \%, \mathrm{p}<0.001)$. Furthermore, right-sided $\mathrm{sCRLM}$ showed significantly shorter cancer specific survival (CSS) than those from left-side $(p<0.001)$. After Cox hazard regression analysis, right-sided PTL still remained to be a strong independent predictor for poor prognosis in this cohort of $s C R L M$ patients (OS, $\mathrm{HR}=1.75,95 \% \mathrm{Cl} 1.34-2.29 ; \mathrm{CSS}, \mathrm{HR}=1.76,95 \% \mathrm{Cl}$ 1.33-2.35). In conclusion, according to this population-based cohort from the SEER database, PTL was a critical prognostic factor that affect long-term OS and CSS in patients with sCRLM after surgical treatment of primary tumor and liver metastases.
\end{abstract}

Key words: synchronous, colorectal liver metastases, primary tumor location, surgical treatment, prognosis

\section{Introduction}

Colorectal cancer (CRC) is the third most common cancer type and the fourth leading cause of cancer-related deaths in the world. Traditionally, CRC was more prevalent in developed countries, while its incidence and mortality has been also on the rise in developing countries over recent decades $(1,2)$. Therefore, many efforts have been devoted in recent years to improve the efficacy of clinical treatment of CRC. However, great histological and molecular heterogeneity has become a major obstacle for effective prognostication and treatment stratification, raising challenges for clinical management. Recent studies have proposed that a significant part of this heterogeneity is captured by the anatomical location 
of the tumor.

From the perspective of embryologic development, the right- and left-sided colon have different developmental origin. The right colon arises from the midgut and the left colon from the hindgut, which are exposed to different luminal environment(3). Accordingly, right- and left-sided CRC differs in demographical and clinical features. Furthermore, genetic studies have revealed differential gene expression patterns and gene mutation landscape between rightand left-sided colon cancers $(4,5)$. For these biological and molecular distinctions, the prognostic value of primary tumor location (PTL) in CRC has been proposed and attracted much attention especially in recent 10 years. On the whole, right-sided tumors was associated with a worse prognosis than left-sided tumors irrespective of tumor stages from several population-based studies worldwide(6-10). However, evidences from these studies also suggested that the prognostic value of PTL seemed to be tumor stage specific. In detail, for early-stage stage (I-II) CRC, similar prognosis was found between right-sided and left-sided CRCs $(11,12)$. For stage III CRC, it was found that right-sided tumors begin to show significantly worse prognosis than left-sided CRCs(11, 13). Then for unresectable stage IV CRC (mCRC), existing results supported that right-sided PTL was also associated with higher mortality regardless of chemotherapy alone or in combination with targeted therapy (BT) (eg, bevacizumab or cetuximab) (14-19). More recently, the role of PTL in predicting response to anti-EGFR based therapy in mCRC became a hotspot. In patients with wild-type KRAS tumors, treatment with cetuximab may possibly benefit only those with left-sided $\mathrm{mCRC}$ but not right-sided cases as recent researches have indicated.(20-25). These evidences supports the conclusion that patients with left-sided RAS wild-type mCRC should be preferentially treated with an anti-EGFR antibody, while in right-sided $\mathrm{mCRC}$, anti-EGFR therapy is not recommended.

As a unique clinical phase of $\mathrm{mCRC}$, synchronous colorectal liver metastasis (sCRLM) has attracted more and more attention. In recent years, the development of treatment concept and surgical techniques has led to revolutionary changes in clinical management of sCRLM, and it is generally accepted that surgical resection of both primary tumor and liver metastases is the only curative treatment strategy for sCRLM. However, the resection for cure is performed significantly less often in cases of sCRLM than for metachronous metastases cases $(6.3 \%$ vs $16.9 \%$, respectively), and the 5-year survival rates were lower with synchronous than with metachronous cases (3.3\% vs $6.1 \%$, respectively)(26).
To date, studies concerning the prognostic value of PTL in SCRLM after resection are limited, especially in those who have metastases confined to the liver. The existing studies were mostly based on single center experience, and all of them both included synchronous and metachronous CRLM cases for study(27-31). Thus, it remains unclear whether the prognosis of sCRLM with right-sided PTL is different from left-sided cases. Here we used population-based data from the SEER database, to analyze the association between PTL and prognostic survival especially in sCRLM after surgical treatment.

\section{Methods}

\section{Data source}

Data was obtained from the Surveillance, Epidemiology, and End Results (SEER) database. The current SEER database consists of 18 populationbased cancer registries that cover approximately $28 \%$ of cancer cases in the United States. This database contains no personal identifiers and is publicly available for cancer studies. And this study was also approved by our institutional review board at Huashan Hospital, Fudan University.

\section{Cohort definition}

We used the National Cancer Institute SEER*Stat software (Version 8.3.2, http://seer.cancer.gov/seer stat) to identify patients. Firstly, patients with CRC were identified with the following inclusion criteria: (1) International Classification of Diseases for Oncology (ICD-O) code 8936; (2) the year of diagnosis ranged from 2010 to 2014; (3) diagnosis was confirmed by histology; (4) aged 18 or older; (5) the cause of death and survival time were both known. Then a stringent exclusive procedure was performed to identify CRC cases with liver-limited metastases. The detailed procedure for patient selection is outlined in Figure 1.

\section{Parameters}

The following parameters were extracted from the SEER database, including sex, age, race, grade, year of diagnosis, marital status, insurance status, primary tumor location, histological type, grade, primary tumor size, neural invasion, lymph node status, tumor deposit and resection margin. The main parameter of interest was each patient's PTL at diagnosis. The division in the right- and left-sided CRC is based on its embryological origin. The embryological border between both parts of the colon is located at the proximal two-thirds of the transverse colon. However, most researchers use the splenic flexure as the demarcation line between right- and left-sided tumors (32). In our study, the right-sided 
CRC includes those originate from caecum, ascending colon, hepatic flexure and transverse colon, while the left part consists of the splenic flexure, descending colon, sigmoid and rectum.

The main outcomes were 5-year overall survival (OS) and cancer specific survival (CSS). CSS was defined from the date of diagnosis to the date of cancer specific death. In this study, CSS was defined as death due to sCRLM, excluding other causes of death.

\section{Statistical analysis}

Clinicopathological parameters were analyzed by chi-square $(x 2)$ test. Kaplan-Meier plots were used to show OS and CSS, and the differences were analyzed by log-rank test. Multivariate Cox proportional hazard models were adopted to analyze the risk factors of survival outcomes. All data analyses were performed using statistical software package SPSS (version 19.0, Inc, Chicago, IL, USA). All statistical tests were evaluated using a two tailed $95 \%$ confidence interval (CI), and a two-side P value of less than 0.05 was set for statistical significance.

\section{Results}

\section{Patient enrollment, selection and characteristics of the study cohort}

In the initial enrollment stage, 22102 patients diagnosed as CRC from 2010 to 2014 were included. Then we conducted a stringent exclusion procedure as shown in Figure 1. We further grouped the patients based on information about metastasis, primary lesion and surgery as stated in Figure 1. Ultimately, 1508 patients in cohort A were selected for our study. Demographics of the 1508 subjects included in the study are listed in Table 1. The median age of the 1508 selected subjects was 61 year old (19-97), and most of them were white $(75.9 \%, n=1145)$ and male $(57.8 \%$, $n=872)$. On pathological types, the majority of patients were adenocarcinoma $(93.6 \%, n=1412)$. Of subjects with known lymph nodal status, most had N1 lymph node involvement (44.2\%), with $19.1 \%$ having N0 and $36.0 \%$ having N2 disease. With respect to PTL, 593 (39.3\%) had right-sided tumors and 915 (60.7\%) had left sided tumors, including 358 (23.7\%) rectal cancer.

\section{Comparison of clinicopathological features between right-sided and left-sided CRC with synchronous liver-limited metastases}

Then we compared the clinicopathological features between right-sided and left-sided sCRLM in cohort A cases. As shown in Table 1, patients originating from right-sided tumors showed different baseline characteristics from left-sided cases in gender (male, $51.8 \%$ vs. $61.7 \%$, $\mathrm{p}<0.001$ ), age ( $\geq 60$ year, $65.3 \%$ vs. $48.3 \%$, $\mathrm{p}<0.001$ ), race (black, $18.7 \%$ vs. $11.1 \%$, $\mathrm{p}<0.001$ ) and marital status (widowed, $13.3 \%$ vs. $6.2 \%$, p $<0.001$ ). In clinicopathological parameters, the right-sided tumors showed significantly higher proportion of poor differentiation or undifferentiated $(30.5 \%$ vs. $15.1 \%, \mathrm{p}<0.001)$, mucinous change $(7.3 \%$ vs. $4.0 \%, \mathrm{p}=0.005), \mathrm{T} 4$ ( $31.3 \%$ vs. $20.1 \%, \mathrm{p}<0.001)$ and N2 $(42.5 \%$ vs. $31.8 \%, p<0.001)$ status, but lower occurrence of neural invasion $(22.1 \%$ vs. $28.5 \%, \mathrm{p}=0.009$ ), compared with originally left-sided tumors. Meanwhile, more lymph nodes (LN) were examined perioperatively in cases of right-sided SCRLM compared with left-sided cases (>12 LN, $88.7 \%$ vs. $81.0 \%$, p $<0.001)$. Otherwise, the two subgroups did not show significant differences in proportion of CEA positive, resection margin or tumor deposits.

Figure 1. Flowchart of patient inclusion in this cohort study. 
Table 1. Baseline clinicopathological characteristics of patients with differed sCRLM locations in SEER database

\begin{tabular}{|c|c|c|c|c|}
\hline \multirow[b]{2}{*}{ Characteristic } & \multicolumn{3}{|c|}{ Primary tumor location } & \multirow[b]{2}{*}{ P-value } \\
\hline & $\begin{array}{l}\text { All Patients } \\
(n=1508)\end{array}$ & $\begin{array}{l}\text { Right } \\
(n=593)\end{array}$ & $\begin{array}{l}\text { Left } \\
(n=915)\end{array}$ & \\
\hline \multicolumn{5}{|l|}{ Patient Related } \\
\hline \multicolumn{5}{|l|}{ Sex } \\
\hline Female & $636(42.2 \%)$ & $286(48.2 \%)$ & $350(38.3 \%)$ & $<0.001$ \\
\hline Male & $872(57.8 \%)$ & $307(51.8 \%)$ & $565(61.7 \%)$ & \\
\hline \multicolumn{5}{|l|}{ Age at diagnosis (years) } \\
\hline median/mean & $61 / 60.9$ & & & \\
\hline$<50$ & $285(18.9 \%)$ & $77(13.0 \%)$ & $208(22.7 \%)$ & $<0.001$ \\
\hline $50-59$ & $395(26.2 \%)$ & $129(21.8 \%)$ & $266(29.1 \%)$ & \\
\hline $60-69$ & $440(29.2 \%)$ & $183(30.9 \%)$ & $257(28.1 \%)$ & \\
\hline $70-79$ & $266(17.6 \%)$ & $124(20.9 \%)$ & $142(15.5 \%)$ & \\
\hline$\geq 80$ & $122(8.1 \%)$ & $80(13.5 \%)$ & $42(4.7 \%)$ & \\
\hline \multicolumn{5}{|l|}{ Race } \\
\hline White & $1145(75.9 \%)$ & $446(75.2 \%)$ & $699(76.4 \%)$ & $<0.001$ \\
\hline Black & $213(14.1 \%)$ & $111(18.7 \%)$ & $102(11.1 \%)$ & \\
\hline Other & $148(9.8 \%)$ & $36(6.1 \%)$ & $112(12.2 \%)$ & \\
\hline Unknow & $2(0.1 \%)$ & $0(0 \%)$ & $2(0.2 \%)$ & \\
\hline Marital status & & & & $<0.001$ \\
\hline Married & $904(59.9 \%)$ & $340(57.3 \%)$ & $564(61.6 \%)$ & \\
\hline Widowed & $136(9.0 \%)$ & $79(13.3 \%)$ & $57(6.2 \%)$ & \\
\hline $\begin{array}{l}\text { Single, separated, or } \\
\text { divorced }\end{array}$ & $400(26.5 \%)$ & $149(25.1 \%)$ & $251(27.4 \%)$ & \\
\hline Unknown & $68(4.5 \%)$ & $25(4.2 \%)$ & $43(4.7 \%)$ & \\
\hline Insurance status & & & & 0.029 \\
\hline Insured & $1253(83.1 \%)$ & $509(85.8 \%)$ & $744(81.3 \%)$ & \\
\hline Any Medicaid & $178(11.8 \%)$ & $54(9.1 \%)$ & $124(13.6 \%)$ & \\
\hline Uninsured & $57(3.8 \%)$ & $24(4.0 \%)$ & $33(3.6 \%)$ & \\
\hline unknown & $20(1.3 \%)$ & $6(1.0 \%)$ & $14(1.5 \%)$ & \\
\hline \multicolumn{5}{|l|}{ Disease Related } \\
\hline CEA level & & & & 0.623 \\
\hline Positive/elevated & $840(55.7 \%)$ & $317(53.5 \%)$ & $523(57.2 \%)$ & \\
\hline Negative/normal & $287(19.0 \%)$ & $113(19.1 \%)$ & $174(19.0 \%)$ & \\
\hline Unknown/Borderline & $381(25.3 \%)$ & $163(27.5 \%)$ & $218(23.8 \%)$ & \\
\hline \multicolumn{5}{|c|}{ Primary tumor size (mm) } \\
\hline$\leq 50$ & $777(51.5 \%)$ & $301(50.8 \%)$ & $510(55.6 \%)$ & 0.003 \\
\hline$>50$ & $408(27.1 \%)$ & $270(45.5 \%)$ & $332(36.3 \%)$ & \\
\hline Tumor deposits & & & & 0.27 \\
\hline Yes & $393(26.1 \%)$ & $146(24.6 \%)$ & $247(27.0 \%)$ & \\
\hline No & $1036(68.7 \%)$ & $418(70.5 \%)$ & $618(67.5 \%)$ & \\
\hline Unknown & $79(5.2 \%)$ & $29(4.9 \%)$ & $50(5.5 \%)$ & \\
\hline \multicolumn{4}{|c|}{ Tumor differentiation(grade) } & $<0.001$ \\
\hline Undifferentiated (IV) & $68(4.5 \%)$ & $40(6.7 \%)$ & $28(3.1 \%)$ & \\
\hline Poor (III) & $251(16.6 \%)$ & $141(23.8 \%)$ & $110(12.0 \%)$ & \\
\hline Moderate (II) & $1072(71.1 \%)$ & $373(62.9 \%)$ & $699(76.4 \%)$ & \\
\hline Well (I) & $40(2.7 \%)$ & $15(2.5 \%)$ & $25(2.7 \%)$ & \\
\hline Unknown & $77(5.1 \%)$ & $24(4.0 \%)$ & $53(5.8 \%)$ & \\
\hline \multicolumn{4}{|l|}{ Histological subtype } & 0.005 \\
\hline Adenocarcinoma & $1412(93.6 \%)$ & $540(91.1 \%)$ & $872(95.3 \%)$ & \\
\hline $\begin{array}{l}\text { Mucinous } \\
\text { adenocarcinoma }\end{array}$ & $80(5.3 \%)$ & $43(7.3 \%)$ & $37(4.0 \%)$ & \\
\hline Other & $16(1.1 \%)$ & $10(1.7 \%)$ & $6(0.7 \%)$ & \\
\hline \multicolumn{4}{|l|}{ Neural invasion } & 0.009 \\
\hline Yes & $392(26.0 \%)$ & $131(22.1 \%)$ & $261(28.5 \%)$ & \\
\hline No & $991(65.7 \%)$ & $407(68.6 \%)$ & $584(63.8 \%)$ & \\
\hline Unknown & $125(8.3 \%)$ & $55(9.3 \%)$ & $70(7.7 \%)$ & \\
\hline \multicolumn{4}{|l|}{ Resection Margin } & 0.484 \\
\hline R0 & $993(65.8 \%)$ & $387(65.3 \%)$ & $606(66.2 \%)$ & \\
\hline R1 & $140(9.3 \%)$ & $59(9.9 \%)$ & $81(8.9 \%)$ & \\
\hline Unknown & $375(24.9 \%)$ & $147(24.8 \%)$ & $228(24.9 \%)$ & \\
\hline \multicolumn{4}{|l|}{ T status } & $<0.001$ \\
\hline $\mathrm{T} 1$ & $24(1.6 \%)$ & $3(0.5 \%)$ & $21(2.3 \%)$ & \\
\hline $\mathrm{T} 2$ & $62(4.1 \%)$ & $24(4.0 \%)$ & $38(4.2 \%)$ & \\
\hline T3 & $1020(67.6 \%)$ & $372(62.7 \%)$ & $648(70.8 \%)$ & \\
\hline $\mathrm{T} 4 \mathrm{a}$ & $274(18.2 \%)$ & $133(22.4 \%)$ & $141(15.4 \%)$ & \\
\hline $\mathrm{T} 4 \mathrm{~b}$ & $96(6.4 \%)$ & $53(8.9 \%)$ & $43(4.7 \%)$ & \\
\hline
\end{tabular}

\begin{tabular}{lllll}
\hline \multirow{2}{*}{ Characteristic } & \multicolumn{3}{l}{ Primary tumor location } & \\
\cline { 2 - 4 } & $\begin{array}{l}\text { All Patients } \\
\text { (n=1508) }\end{array}$ & $\begin{array}{l}\text { Right } \\
\text { (n=593) }\end{array}$ & $\begin{array}{l}\text { Left } \\
\text { (n=915) }\end{array}$ & \\
\hline Unknown & $32(2.1 \%)$ & $8(1.3 \%)$ & $24(2.6 \%)$ & \\
Nodal status & & & & $<0.001$ \\
NX & $11(0.7 \%)$ & $2(0.3 \%)$ & $9(1.0 \%)$ & \\
N0 & $288(19.1 \%)$ & $93(15.7 \%)$ & $195(21.3 \%)$ & \\
N1 & $666(44.2 \%)$ & $246(41.5 \%)$ & $420(45.9 \%)$ & \\
N2 & $543(36.0 \%)$ & $252(42.5 \%)$ & $291(31.8 \%)$ & \\
Adequate lymph node examination (i.e., 12 nodes) & & $<0.001$ \\
No & $224(14.9 \%)$ & $58(9.8 \%)$ & $166(18.1 \%)$ & \\
Yes & $1267(84.0 \%)$ & $526(88.7 \%)$ & $741(81.0 \%)$ & \\
Unknown & $17(1.1 \%)$ & $9(1.5 \%)$ & $8(0.9 \%)$ & \\
\hline
\end{tabular}

\section{Survival analysis based on primary tumor location}

To determine whether the primary tumor location is associated with distinct clinical outcomes in sCRLM, we then evaluated the prognosis of the two subsite in our cohort. Of note, Kaplan-Meier plots of 5 -year CSS were significantly worse in patients with right-sided sCRLM (Figure 2, p<0.001), and left-sided colon cancer and rectal cancer showed similarly better CSS (Figure 3, p <0.001). Consistently, Kaplan-Meier survival curve based on more detailed tumor subsite location are presented in Supplementary Figure 1. Splenic flexure, sigmoid colon and rectal cases had the highest overall survival estimates, descending and transverse colon cancers had intermediate survival estimates, whereas proximal cancers had the poorest survival.

\section{Multivariate analysis of factors associated with Mortality}

Next, we evaluated the association of clinicopathological factors with the outcome of sCRLM patients. On multivariable analyses after controlling for competing risk factors, several factors remained independently associated with OS and CSS, including old age $(\geq 80)(\mathrm{OS}, \mathrm{HR}=3.5,95 \%$ CI 2.04-6.02; CSS, $\mathrm{HR}=3.31,95 \% \mathrm{CI} 1.87-5.87)$, N2 status (OS, $\mathrm{HR}=1.54$, 95\% CI 1.02-2.31; CSS, HR=1.73, 95\% CI 1.11-2.71), resection margin (OS, $\mathrm{HR}=1.83,95 \%$ CI 1.29-2.59; CSS, HR $=1.89,95 \%$ CI 1.32-2.71), as well as right-sided PTL (OS, HR=1.75, 95\% CI 1.34-2.29; CSS, HR=1.76, 95\% CI 1.33-2.35) (all $\mathrm{P}<0.05)$. While other factors such as gender, race, marital status, insurance status, CEA positive, tumor size, tumor deposit, histological type, differentiation, neural invasion and $\mathrm{T}$ status were not correlated with OS or CSS in this cohort (Table 2).

\section{Discussion}

During the last years, the impact of PTL in CRC has been intensively studied by multiple research groups. Now it has been recognized that the rightand left-sided CRC have different developmental origin and correspondingly diverse oncogenic 
mechanism, contributing to side specific clinical outcome and therapeutic response $(4,5,32)$. However, whether this prognostic distinction also exists in specific clinical stages of CRC remains obscure. In this study, using the SEER database, we confirmed the emerging notion that in stage IVA CRC with liver-limited metastases, right-sided PTL also predict worse prognosis than left-sided tumors, for cases who accepted surgical treatment of both primary and hepatic metastases.

The mechanism that contributed to the great prognostic discrepancy between right- and left-sided CRC has been intensively investigated. Demographically, right-sided tumors differ in gender, age and race compared to left-sided cases. Furthermore, CRC in patients over 80 s are far more likely to be in the right-sided colon, as is shown in our study. Clinically, right-sided sCRLM appears to have an intent of low differentiated degrees, larger size of primary lesion and early lymph nodes invasion, indicating its relative higher malignancy degrees, which can also partly explain its negative significance in prognosis. Biologically, the differences in luminal content and bacterial flora between the right- and left-sided colon may also influence tumor biology and prognosis(32). Additionally on molecular level, right-sided carcinomas are more often KRAS/BRAF-mutated, more enriched in microsatellite instability (MSI), CpG island methylation phenotype (CIMP) and CMS1 molecular subtype. Left-sided tumors more often harbor chromosomal instability (CIN) and CMS2 molecular subtype $(5,32)$. Based these evidences, it is convincingly suggested that the above clinical and molecular parameters converged to contribute to the prognostic distinction between right- and left-sided sCRLM.

In recent three years, there have been several studies focusing on the association between PTL and prognostic survival in CRLM after surgical treatment based on single center data. In 2016, Sasaki K from Johns Hopkins Hospital firstly reported the prognostic implications of PTL in CRLM undergoing resection. Of note, patients with left-sided primary tumor had a shorter RFS compared with patients who had a right-sided tumor, while right-sided primary CRC tumors had a shorter OS compared with left-sided tumors(27). Interestingly, their subsequent study revealed a differential prognostic implication of KRAS status after hepatectomy for CRLM according to PTL. Among patients with a right-sided CRC, 5-year RFS and OS were not correlated with KRAS status. In contrast, among patients from a left-sided primary CRC, 5-year RFS and OS were worse among patients with mutant-type KRAS(33). Similarly, experience from MD Anderson Cancer Center found that both RFS and OS after hepatic resection were worse in patients with midgut origin tumors (right-sided)(28). Then data from MSKCC showed that right-sided CRLM had significantly worse OS compared to left-sided CRLM after resection, while the median RFS was only marginally different(29). Consistently, a study from Europe found that right-sided PTL is associated with worse OS after surgery for CLM, but seems to have no influence on PFS(30). However, two recent study from Brazil and China reported that PTL did not affect OS or DFS for patients with resected $\operatorname{CRLM}(31,34)$. All the above studies in combination with our study indicate that PTL is an important prognostic factor for OS, while its role in RFS/PFS/DFS is still controversial.

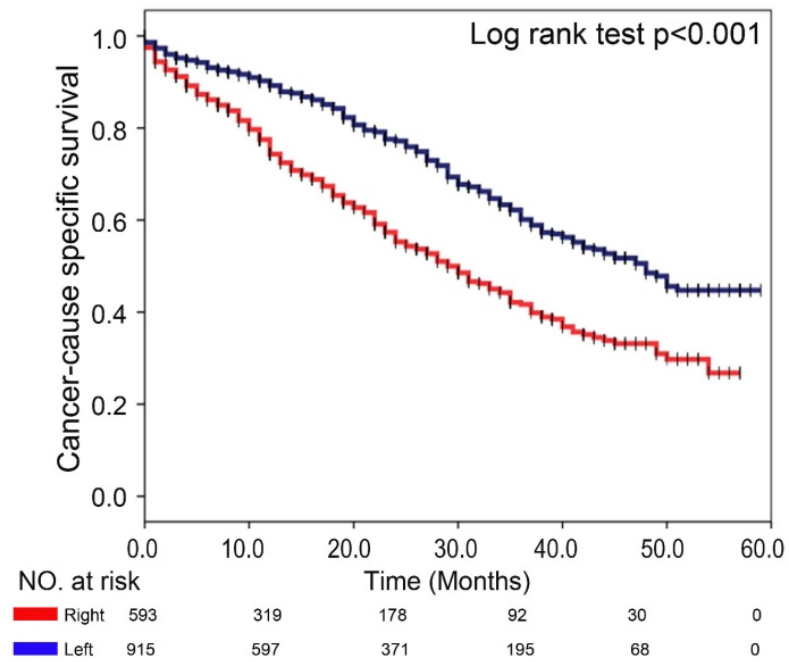

Figure 2. Comparison of CSS between left- and right-sided sCRLM patients in Kaplan-Meier survival analysis.

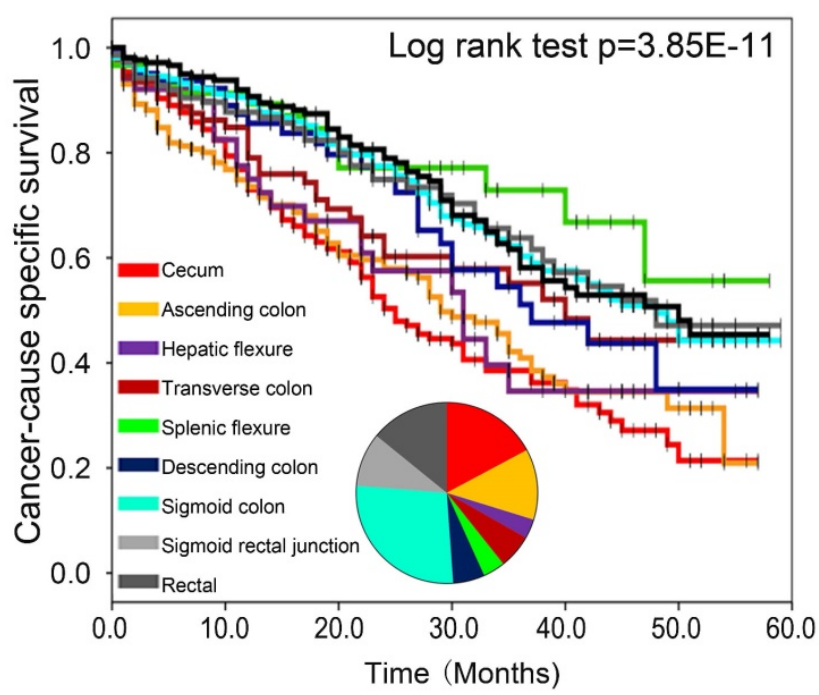

Figure 3. Comparison of CSS among right, left and rectal sCRLM patients in Kaplan-Meier survival analysis. 
Table 2. Survival analysis of subgroups based on clinicopathological characteristics

\begin{tabular}{|c|c|c|c|c|c|c|}
\hline \multirow[t]{2}{*}{ Variable } & \multicolumn{3}{|l|}{ Overall Survival } & \multicolumn{3}{|c|}{ Cancer-Specific Survival } \\
\hline & No./Total No. (\%) & Hazard ratio $(95 \% \mathrm{CI})$ & p value & No./Total No. (\%) & Hazard ratio $(95 \% \mathrm{CI})$ & p-value \\
\hline \multicolumn{7}{|l|}{ Sex } \\
\hline female & $247 / 21(36.0 \%)$ & 1 [Reference] & & $232 / 21(37.9 \%)$ & 1 [Reference] & \\
\hline male & $328 / 32(32.6 \%)$ & $0.85(0.65-1.11)$ & 0.232 & $292 / 42(38.1 \%)$ & $0.79(0.60-1.05)$ & 0.104 \\
\hline \multicolumn{7}{|l|}{ Race } \\
\hline White & $428 / 34(34.9 \%)$ & 1 [Reference] & & $390 / 34(39.0 \%)$ & 1 [Reference] & \\
\hline Black & $100 / 10(26.6 \%)$ & $1.11(0.77-1.59)$ & 0.581 & $91 / 10(29.9 \%)$ & $1.07(0.73-1.57)$ & 0.741 \\
\hline Other & $47 / 8(39.3 \%)$ & $1.08(0.68-1.69)$ & 0.756 & $43 / 8(42.0 \%)$ & $0.99(0.61-1.61)$ & 0.972 \\
\hline \multicolumn{7}{|l|}{ Age } \\
\hline$<50$ & $81 / 10(40.9 \%)$ & 1 [Reference] & & $77 / 10(41.8 \%)$ & 1 [Reference] & \\
\hline $50-59$ & $124 / 29(41.0 \%)$ & $0.77(0.48-1.22)$ & 0.266 & $116 / 29(42.7 \%)$ & $0.76(0.47-1.24)$ & 0.271 \\
\hline $60-69$ & $165 / 23(35.2 \%)$ & $1.29(0.85-1.93)$ & 0.23 & $149 / 23(40.2 \%)$ & $1.30(0.85-2.00)$ & 0.226 \\
\hline $70-79$ & $124 / 10(27.6 \%)$ & $1.89(1.22-2.92)$ & 0.004 & $111 / 14(35.6 \%)$ & $1.86(1.18-2.96)$ & 0.008 \\
\hline$\geq 80$ & $81 / 1(7.6 \%)$ & $3.5(2.04-6.02)$ & $<0.001$ & $71 / 1(9.6 \%)$ & $3.31(1.87-5.87)$ & $<0.001$ \\
\hline \multicolumn{7}{|l|}{ Marital status } \\
\hline Married & $306 / 53(38.7 \%)$ & 1 [Reference] & & $277 / 53(42.5 \%)$ & 1 [Reference] & \\
\hline Widowed & $77 / 9(23.0 \%)$ & $0.89(0.56-1.41)$ & 0.612 & $69 / 9(26.7 \%)$ & $0.86(0.53-1.39)$ & 0.531 \\
\hline Single/Separated/divorced & $167 / 13(30.1 \%)$ & $1.27(0.94-1.71)$ & 0.127 & $156 / 13(33.5 \%)$ & $1.26(0.92-1.73)$ & 0.155 \\
\hline \multicolumn{7}{|l|}{ Insurance status } \\
\hline Uninsured & $24 / 5(31.4 \%)$ & 1 [Reference] & & $23 / 5(32.2 \%)$ & 1 [Reference] & \\
\hline Insured & $470 / 37(34.8 \%)$ & $0.66(0.35-1.23)$ & 0.192 & $425 / 37(39.0 \%)$ & $0.65(0.34-1.25)$ & 0.197 \\
\hline Any Medicaid & $76 / 9(30.3 \%)$ & $1.15(0.57-2.35)$ & 0.694 & $71 / 9(32.3 \%)$ & $1.20(0.57-2.53)$ & 0.631 \\
\hline \multicolumn{7}{|l|}{ CEA level } \\
\hline Negative & $83 / 21(51.3 \%)$ & 1 [Reference] & & $78 / 21(52.8 \%)$ & 1 [Reference] & \\
\hline Positive & $333 / 23(29.4 \%)$ & $1.38(0.99-1.92)$ & 0.056 & $300 / 23(34.3 \%)$ & $1.37(0.97-1.93)$ & 0.078 \\
\hline \multicolumn{7}{|l|}{ Tumor size } \\
\hline$<50$ & $238 / 19(35.1 \%)$ & 1 [Reference] & & $218 / 19(37.5 \%)$ & 1 [Reference] & \\
\hline$\geq 50$ & $303 / 37(31.6 \%)$ & $1.11(0.86-1.44)$ & 0.431 & $278 / 37(35.3 \%)$ & $1.17(0.89-1.54)$ & 0.251 \\
\hline \multicolumn{7}{|l|}{ Tumor deposits } \\
\hline Negative & $361 / 35(38.6 \%)$ & 1 [Reference] & & $326 / 35(42.5 \%)$ & 1 [Reference] & \\
\hline Positive & $169 / 13(27.6 \%)$ & $1.30(0.98-1.73)$ & 0.071 & $160 / 13(29.9 \%)$ & $1.31(0.97-1.77)$ & 0.074 \\
\hline \multicolumn{7}{|l|}{ Histological subtype } \\
\hline Mucinous adenocarcinoma & $34 / 4(27.8 \%)$ & 1 [Reference] & & $32 / 4(32.0 \%)$ & 1 [Reference] & \\
\hline Adenocarcinoma & $534 / 42(34.4 \%)$ & $1.13(0.64-1.98)$ & 0.68 & $485 / 42(38.4 \%)$ & $1.14(0.63-2.06)$ & 0.663 \\
\hline \multicolumn{7}{|l|}{ Differentiation } \\
\hline Well differentiated & $16 / 7(37.0 \%)$ & 1 [Reference] & & $15 / 7(39.5 \%)$ & 1 [Reference] & \\
\hline Moderately differentiated & $372 / 32(36.0 \%)$ & $1.16(0.52-2.59)$ & 0.711 & $334 / 32(40.5 \%)$ & $1.01(0.45-2.26)$ & 0.984 \\
\hline Poorly differentiated & $131 / 9(24.3 \%)$ & $2.03(0.86-4.78)$ & 0.106 & $123 / 9(26.9 \%)$ & $1.81(0.76-4.32)$ & 0.179 \\
\hline Undifferentiated & $37 / 4(18.2 \%)$ & $1.79(0.69-4.67)$ & 0.235 & $34 / 4(20.6 \%)$ & $1.58(0.60-4.20)$ & 0.357 \\
\hline \multicolumn{7}{|l|}{ Neural invasion } \\
\hline Negative & $352 / 29(37.3 \%)$ & 1 [Reference] & & $320 / 29(40.4 \%)$ & 1 [Reference] & \\
\hline Positive & $161 / 19(28.6 \%)$ & $1.063(0.79-1.43)$ & 0.684 & $149 / 19(31.3 \%)$ & $1.08(0.80-1.48)$ & 0.615 \\
\hline \multicolumn{7}{|l|}{ T status(no/yes) } \\
\hline $\mathrm{T} 1$ & $7 / 9(57.0 \%)$ & 1 [Reference] & & $6 / 9(62.7 \%)$ & 1 [Reference] & \\
\hline $\mathrm{T} 2$ & $17 / 11(49.7 \%)$ & $0.73(0.09-5.91)$ & 0.766 & $16 / 11(52.4 \%)$ & $0.58(0.07-4.76)$ & 0.608 \\
\hline $\mathrm{T} 3$ & $361 / 35(36.9 \%)$ & $0.87(0.12-6.38)$ & 0.888 & $320 / 35(41.8 \%)$ & $0.67(0.09-4.99)$ & 0.699 \\
\hline $\mathrm{T} 4$ & $180 / 10(21.9 \%)$ & $0.81(0.11-6.06)$ & 0.834 & $172 / 10(23.2 \%)$ & $0.69(0.09-5.18)$ & 0.714 \\
\hline \multicolumn{7}{|l|}{ Nodal status } \\
\hline No & $83 / 40(52.4 \%)$ & 1 [Reference] & & $73 / 44(57.1 \%)$ & 1 [Reference] & \\
\hline N1 & $240 / 16(33.2 \%)$ & $1.23(0.84-1.81)$ & 0.296 & $218 / 16(36.0 \%)$ & $1.37(0.90-2.09)$ & 0.146 \\
\hline N2 & $249 / 24(26.5 \%)$ & $1.54(1.02-2.31)$ & 0.04 & $230 / 30(30.8 \%)$ & $1.73(1.11-2.71)$ & 0.015 \\
\hline \multicolumn{7}{|l|}{ Resection Margin } \\
\hline Negative & $343 / 29(35.2 \%)$ & 1 [Reference] & & $303 / 29(39.8 \%)$ & 1 [Reference] & \\
\hline Positive & $82 / 10(21.2 \%)$ & $1.83(1.29-2.59)$ & 0.001 & $79 / 10(21.9 \%)$ & $1.89(1.32-2.71)$ & $<0.001$ \\
\hline Primary tumor location & & & & & & \\
\hline Left-sided & $289 / 55(40.1 \%)$ & 1 [Reference] & & $261 / 59(44.8 \%)$ & 1 [Reference] & \\
\hline Right-sided & $286 / 9(24.6 \%)$ & $1.75(1.34-2.29)$ & $<0.001$ & $263 / 9(26.8 \%)$ & $1.76(1.33-2.35)$ & $<0.001$ \\
\hline
\end{tabular}

Traditionally, the clinical prognostication of sCRLM after surgical treatment has long been relegated to clinical staging/scoring system(35-39) or nomograms(40). A major clinically relevant finding of this study is that prognostication of sCRLM based on PTL may be predictive of prognosis (OS and RFS) and is clinically applicable. Our results indicated that adding PTL may provide an important optimization for currently used sCRLM prognosis prediction models.

Compared to the published studies including both synchronous and metachronous CRLM, our present study mainly focused on sCRLM, which 
further reduced the heterogeneity. However, the limitations of our study are obvious for its nature of retrograde cohort study. Primarily, all the patients enrolled were diagnosed between 2010 and 2014 from diverse medical institutions, which means there is no standard evaluation on surgical resections, providing a confounding factor. Moreover, information about the liver metastases retrieved from the SEER database is limited, such as the number and largest size of liver lesions.

Conclusively, for sCRLM patients underwent surgical resection on both primary tumor and liver metastases, PTL can act as an effectively predictive indicator for prognosis. In the future, selectively prognostic and therapeutic stratification may be suggested in clinical practice for right- and left-sided sCRLM.

\section{Supplementary Material}

Supplementary figures.

http://www.jcancer.org/v10p1593s1.pdf

\section{Acknowledgements}

This study was supported by China National Key Projects for Infectious Disease (No.2017ZX102032 07), Shanghai Sailing Program (No.17YF1401600) and the NSFC General Program (No. 81672820, No. 81472677 and No.81672365).

\section{Competing Interests}

The authors have declared that no competing interest exists.

\section{References}

1. Jemal A, Bray F, Center MM, Ferlay J, Ward E, Forman D. Global cancer statistics. CA Cancer J Clin. [Journal Article]. 2011;61(2):69-90.

2. Torre LA, Bray F, Siegel RL, Ferlay J, Lortet-Tieulent J, Jemal A. Global cancer statistics, 2012. CA Cancer J Clin. [Journal Article]. 2015;65(2):87-108.

3. Gervaz P, Bucher P, Morel P. Two colons-two cancers: paradigm shift and clinical implications. J SURG ONCOL. [Journal Article; Review]. 2004;88(4):261-6

4. Stintzing S, Tejpar S, Gibbs P, Thiebach L, Lenz HJ. Understanding the role of primary tumour localisation in colorectal cancer treatment and outcomes. EUR J CANCER. [Journal Article; Review; Research Support, Non-U.S. Gov't]. 2017;84:69-80.

5. Missiaglia E, Jacobs B, D'Ario G, Di Narzo AF, Soneson C, Budinska E, et al. Distal and proximal colon cancers differ in terms of molecular, pathological, and clinical features. ANN ONCOL. [Journal Article; Research Support, Non-U.S. Gov't]. 2014;25(10):1995-2001.

6. Meguid RA, Slidell $\mathrm{MB}$, Wolfgang $\mathrm{CL}$, Chang $\mathrm{DC}$, Ahuja $\mathrm{N}$ Is there a difference in survival between right- versus left-sided colon cancers? ANN SURG ONCOL. [Comparative Study; Journal Article; Research Support, N.I.H., Extramural]. 2008;15(9):2388-94.

7. Suttie SA, Shaikh I, Mullen R, Amin AI, Daniel T, Yalamarthi S. Outcome of right- and left-sided colonic and rectal cancer following surgical resection. COLORECTAL DIS. [Journal Article]. 2011;13(8):884-9.

8. Jess P, Hansen IO, Gamborg M, Jess T. A nationwide Danish cohort study challenging the categorisation into right-sided and left-sided colon cancer. BMJ OPEN. [Journal Article]. 2013;3(5).

9. Nitsche U, Stogbauer F, Spath C, Haller B, Wilhelm D, Friess H, et al. Right Sided Colon Cancer as a Distinct Histopathological Subtype with Reduced Prognosis. Dig Surg. [Comparative Study; Journal Article]. 2016;33(2):157-63.

10. Petrelli F, Tomasello G, Borgonovo K, Ghidini M, Turati L, Dallera P, et al. Prognostic Survival Associated With Left-Sided vs Right-Sided Colon Cancer: A Systematic Review and Meta-analysis. JAMA ONCOL. [Journal Article]. 2016 .
11. Weiss JM, Pfau PR, O'Connor ES, King J, LoConte N, Kennedy G, et al. Mortality by stage for right- versus left-sided colon cancer: analysis of surveillance, epidemiology, and end results--Medicare data. J CLIN ONCOL. [Journal Article; Research Support, N.I.H., Extramural; Research Support, Non-U.S. Gov't]. 2011;29(33):4401-9.

12. Karim S, Brennan K, Nanji S, Berry SR, Booth CM. Association Between Prognosis and Tumor Laterality in Early-Stage Colon Cancer. JAMA ONCOL. [Journal Article]. 2017;3(10):1386-92.

13. Taieb J, Kourie HR, Emile JF, Le Malicot K, Balogoun R, Tabernero J, et al. Association of Prognostic Value of Primary Tumor Location in Stage III Colon Cancer With RAS and BRAF Mutational Status. JAMA ONCOL. [Journal Article]. 2017

14. Ferrand F, Malka D, Bourredjem A, Allonier C, Bouche O, Louafi S, et al. Impact of primary tumour resection on survival of patients with colorectal cancer and synchronous metastases treated by chemotherapy: results from the multicenter, randomised trial Federation Francophone de Cancerologie Digestive 9601. EUR J CANCER. [Clinical Trial, Phase III; Journal Article; Multicenter Study; Randomized Controlled Trial; Research Support, Non-U.S. Gov't]. 2013;49(1):90-7.

15. Modest DP, Schulz C, von Weikersthal LF, Quietzsch D, von Einem JC, Schalhorn A, et al. Outcome of patients with metastatic colorectal cancer depends on the primary tumor site (midgut vs. hindgut): analysis of the FIRE1-trial (FuFIRI or mIROX as first-line treatment). Anticancer Drugs. [Clinical Trial, Phase III; Journal Article; Randomized Controlled Trial]. 2014;25(2):212-8

16. Price TJ, Beeke C, Ullah S, Padbury R, Maddern G, Roder D, et al. Does the primary site of colorectal cancer impact outcomes for patients with metastatic disease? CANCER-AM CANCER SOC. [Journal Article]. 2015;121(6):830-5.

17. Ishihara S, Nishikawa $T$, Tanaka $T$, Tanaka J, Kiyomatsu $T$, Kawai K, et al. Prognostic impact of tumor location in stage IV colon cancer: a propensity score analysis in a multicenter study. INT J SURG. [Journal Article; Multicenter Study]. 2014;12(9):925-30.

18. Loupakis F, Yang D, Yau L, Feng S, Cremolini C, Zhang W, et al. Primary tumor location as a prognostic factor in metastatic colorectal cancer. J Natl Cancer Inst. [Journal Article; Research Support, N.I.H., Extramural; Research Support, Non-U.S. Gov't]. 2015;107(3).

19. Ahmed S, Pahwa P, Le D, Chalchal H, Chandra-Kanthan S, Iqbal N, et al. Primary Tumor Location and Survival in the General Population With Metastatic Colorectal Cancer. Clin Colorectal Cancer. [Journal Article]. 2018;17(2):e201-6.

20. Brule SY, Jonker DJ, Karapetis CS, O'Callaghan CJ, Moore MJ, Wong R, et al. Location of colon cancer (right-sided versus left-sided) as a prognostic factor and a predictor of benefit from cetuximab in NCIC CO.17. EUR J CANCER. [Clinical Trial, Phase III; Journal Article; Randomized Controlled Trial]. 2015;51(11):1405-14.

21. Tejpar S, Stintzing S, Ciardiello F, Tabernero J, Van Cutsem E, Beier F, et al. Prognostic and Predictive Relevance of Primary Tumor Location in Patients With RAS Wild-Type Metastatic Colorectal Cancer: Retrospective Analyses of the CRYSTAL and FIRE-3 Trials. JAMA ONCOL. [Journal Article]. 2016.

22. Arnold D, Lueza B, Douillard JY, Peeters M, Lenz HJ, Venook A, et al. Prognostic and predictive value of primary tumour side in patients with RAS wild-type metastatic colorectal cancer treated with chemotherapy and EGFR directed antibodies in six randomized trials. ANN ONCOL. [Journal Article]. 2017;28(8):1713-29.

23. Boeckx N, Koukakis R, Op DBK, Rolfo C, Van Camp G, Siena S, et al. Primary tumor sidedness has an impact on prognosis and treatment outcome in metastatic colorectal cancer: results from two randomized first-line panitumumab studies. ANN ONCOL. [Journal Article]. 2017;28(8):1862-8.

24. Holch JW, Ricard I, Stintzing S, Modest DP, Heinemann V. The relevance of primary tumour location in patients with metastatic colorectal cancer: A meta-analysis of first-line clinical trials. EUR J CANCER. [Journal Article; Meta-Analysis]. 2017;70:87-98.

25. Aljehani MA, Morgan JW, Guthrie LA, Jabo B, Ramadan M, Bahji K, et al. Association of Primary Tumor Site With Mortality in Patients Receiving Bevacizumab and Cetuximab for Metastatic Colorectal Cancer. JAMA SURG. [Journal Article]. 2018;153(1):60-7.

26. Manfredi S, Lepage C, Hatem C, Coatmeur O, Faivre J, Bouvier AM. Epidemiology and management of liver metastases from colorectal cancer. ANN SURG. [Journal Article]. 2006;244(2):254-9.

27. Sasaki K, Andreatos N, Margonis GA, He J, Weiss M, Johnston F, et al. The prognostic implications of primary colorectal tumor location on recurrence and overall survival in patients undergoing resection for colorectal liver metastasis. J SURG ONCOL. [Journal Article]. 2016;114(7):803-9.

28. Yamashita S, Brudvik KW, Kopetz SE, Maru D, Clarke CN, Passot G, et al. Embryonic Origin of Primary Colon Cancer Predicts Pathologic Response and Survival in Patients Undergoing Resection for Colon Cancer Liver Metastases. ANN SURG. [Journal Article]. 2018;267(3):514-20.

29. Creasy JM, Sadot E, Koerkamp BG, Chou JF, Gonen M, Kemeny NE, et al. The Impact of Primary Tumor Location on Long-Term Survival in Patients Undergoing Hepatic Resection for Metastatic Colon Cancer. ANN SURG ONCOL. [Journal Article]. 2018;25(2):431-8.

30. Dupre A, Malik HZ, Jones RP, Diaz-Nieto R, Fenwick SW, Poston GJ. Influence of the primary tumour location in patients undergoing surgery for colorectal liver metastases. Eur J Surg Oncol. [Journal Article; Multicenter Study; Research Support, Non-U.S. Gov't]. 2018;44(1):80-6. 
31. Marques MC, C RH, Costa WJ, de Jesus V, de Macedo MP, Diniz AL, et al. Is primary sidedness a prognostic factor in patients with resected colon cancer liver metastases (CLM)? J SURG ONCOL. [Journal Article]. 2018;117(5):858-63.

32. Boeckx N, Janssens K, Van Camp G, Rasschaert M, Papadimitriou K, Peeters $\mathrm{M}$, et al. The predictive value of primary tumor location in patients with metastatic colorectal cancer: A systematic review. Crit Rev Oncol Hematol. [Journal Article; Review]. 2018;121:1-10.

33. Sasaki K, Margonis GA, Wilson A, Kim Y, Buettner S, Andreatos N, et al. Prognostic Implication of KRAS Status after Hepatectomy for Colorectal Liver Metastases Varies According to Primary Colorectal Tumor Location. ANN SURG ONCOL. [Journal Article]. 2016;23(11):3736-43.

34. Wang K, Xu D, Yan XL, Poston G, Xing BC. The impact of primary tumour location in patients undergoing hepatic resection for colorectal liver metastasis. Eur J Surg Oncol. [Journal Article]. 2018;44(6):771-7.

35. Fong Y, Fortner J, Sun RL, Brennan MF, Blumgart LH. Clinical score for predicting recurrence after hepatic resection for metastatic colorectal cancer: analysis of 1001 consecutive cases. ANN SURG. [Journal Article; Research Support, U.S. Gov't, P.H.S.]. 1999;230(3):309-18, 318-21.

36. Iwatsuki S, Dvorchik I, Madariaga JR, Marsh JW, Dodson F, Bonham AC, et al. Hepatic resection for metastatic colorectal adenocarcinoma: a proposal of a prognostic scoring system. J Am Coll Surg. [Journal Article]. 1999;189(3):291-9.

37. Rees M, Tekkis PP, Welsh FK, O'Rourke T, John TG. Evaluation of long-term survival after hepatic resection for metastatic colorectal cancer: a multifactorial model of 929 patients. ANN SURG. [Journal Article]. 2008;247(1):125-35.

38. Brudvik KW, Jones RP, Giuliante F, Shindoh J, Passot G, Chung MH, et al. RAS Mutation Clinical Risk Score to Predict Survival After Resection of Colorectal Liver Metastases. ANN SURG. [Journal Article]. 2017.

39. Sasaki K, Morioka D, Conci S, Margonis GA, Sawada Y, Ruzzenente A, et al. The Tumor Burden Score: A New "Metro-ticket" Prognostic Tool For Colorectal Liver Metastases Based on Tumor Size and Number of Tumors. ANN SURG. [Journal Article]. 2018;267(1):132-41.

40. Imai K, Allard MA, Castro BC, Vibert E, Sa CA, Cherqui D, et al. Nomogram for prediction of prognosis in patients with initially unresectable colorectal liver metastases. Br J Surg. [Journal Article]. 2016;103(5):590-9. 